\title{
Modified Self-Organizing Map Algorithm for Brain Tumor Detection and Analysis Using Magnetic Resonance Brain Images
}

\author{
Abd El Kader Isselmou, Guizhi Xu, Shuai Zhang, Sani Saminu \\ Province-Ministry Joint Key Laboratory of Electromagnetic Field and Electrical Apparatus Reliability, \\ Department of Biomedical Engineering, Hebei University of Technology, Tianjin, China. \\ isselmou_kader@yahoo.com
}

\begin{abstract}
medical image processing play an important role to help radiologists and support their decisions in diagnosis of the patient, magnetic resonance imaging (MRI) has ability to diagnosis the small details in the human body with a high resolution; in this paper, we propose modified self-organizing map algorithm (MSOM) for brain tumor detection and analysis using magnetic resonance brain images the significance of the (MSOM) algorithm is ability to detect tumor area in the magnetic resonance brain image (MRI) clearly with a high accuracy and best performance according of different values, the advantage of method proposed can segment and detect different types of MRI brain images FLAIR, T1 and T2-weight images with same performance and accuracy, the (MSOM) method start through input magnetic resonance brain image (MRI) and preprocessing applied to remove the noise from the image, applied modified selforganizing map (MSOM), applied tumor area, performance of the method, finally the applied of modified self-organizing map (MSOM) gave a best results us shown in the results.
\end{abstract}

Keywords: MRI, brain tumor detection, modified self-organizing map, accuracy values.

\section{Introduction}

At present, medical imaging, systems and medical image processing have been revolutionized by new technologies used based on advanced hardware and software architecture. There are many methods of medical imaging systems, all of which create visual representation of the human body and human organs for clinical analysis. Treatment and medical intervention. Because all systems of acquisition and treatment, medical imaging systems sometimes suffer from technical barriers such as device noise, as well as voluntary and involuntary patient movements during medical examination. Moreover, physical limitations are due to radiation sensitivity, magnetic fields and chemical products used [1]. All these factors can make it difficult to interpret and / or analyze this information and medical signals. BMRI, in particular, is one of the most commonly used techniques in imaging to visualize and analyze human head and components. However, identifying and detecting brain abnormalities remains a major challenge and an open search for improved medical diagnosis. Under these circumstances, CAD is very important for improving medical analysis and treatment. Brain tumor is one of the most common brain conditions in children and adults. Brain tumors are the cause of a quarter of cancer deaths in the world. Brain tumor is a group of cells that randomly grow inside or around the brain. There are two classes of tumors, the first 
is a non-cancerous tumor (benign) and the second is a cancerous tumor (malignant). Another abnormality in the brain called brain edema associated with brain tumors is very common and can occur and surround brain tumors [2]. Image processing algorithms and techniques provide tremendous help in this research and provide an additional opinion to improve the analysis and accurate diagnosis of radiologists. In literature, many researchers have proposed different methodologies of BMRI images to achieve the discovery of brain abnormalities with minimal human interaction.

Two fully-unsupervised methods to MR brain image segmentation using SOM-based strategies are recommended by Oritz et al [3] segment T1-weighted images are determined by SOM (Self-Organizing Map) technique. The using the segmentation algorithm is still restricted to process T1-weighted images. Further, the sensitivity value manufactured by the algorithm requires improvement.

Logeswari and Karnan [4] reported brain tumor detection using a segmentation process based on SelfOrganizing Maps (SOM). The segmentation process is curbed to segment T2weighted image sequences.

Logeswari and Karnan [5] offered brain tumor detection using segmentation process based on Hierarchical Self-Organizing Map (HSOM) technique, were T2 weighted images was segmented in an average time of 29.9708 seconds, which requires further minimization.

Yan Li and Zheru et al [6] performed MR brain image segmentation using SOM, which requires a reduction in MSE values.

Segmentation algorithms put forth by Guler et al [7], Ong et al [8], Alirezaie et al [9], Sun [10] and Jiang et al [11] have utilized unsupervised neural network technique namely, self-organizing maps (SOM).

Segmentation of T1-weighted images alone is completed employing an automated algorithm, which mixes stationary wavelets and Ayse Demirhan [12] created Self-Organizing Map (SOM).

In this paper, we proposed modified self-organizing map (MSOM) algorithm has ability to identify the tumor area using MR brain images with a best accuracy than all the methods created using self-organizing map (SOM) algorithm based on different values accuracy, nJaccard, nDice, sensitivity, specificity, recall and precision value.

\section{Materials}

Approximately 15FLAIR, T1.T2-weight brain images, we obtained from the different patients and different Age groups have been used in this paper, from the Tianjin Medical Hospital University details are listed below from patients:

1. The clinical image of the patient from the age of thirty-seven suffering from used meningioma.

2. The clinical image of the patient age of thirty-two suffering from and using high- quality astrocytes.

3. Images of patients with metastatic tumor and bronchial cancer, taken from Nouakchott hospital of oncology and used inventory, figure.1 shown example of different types of MR brain images with tumor. 
Abd El Kader Isselmou, Guizhi Xu, Shuai Zhang, Sani Saminu; Modified Self-Organizing Map Algorithm for Brain Tumor Detection and Analysis Using Magnetic Resonance Brain Images. Journal of Biomedical Engineering and Medical Imaging, Volume 6, No 2, April (2019), pp 27-34

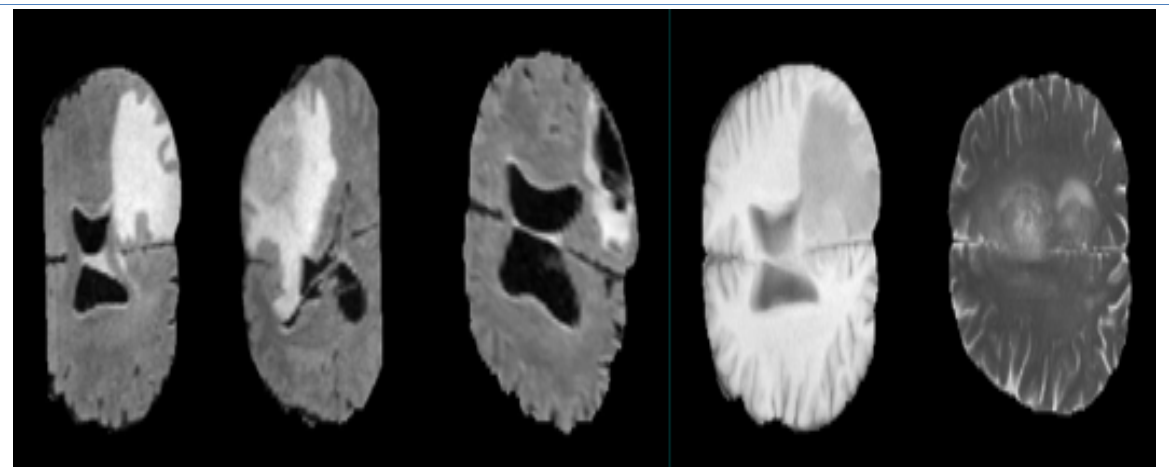

Figure.1. Flair, T1.T1 weight brain images with tumor

\section{Methods}

The methodology in this paper include five necessary stages as follows:

Stage1: Input MR Brain image (original image)

Stage2: pre-processing based on color quantization, tumor masking, and morphological cleaning.

Stage3: segmentation using modified self-organizing map (MSOM) algorithm

Stage4: detect tumor area or detect tumor alone in MR brain image

Stage5: analysis stage based on performance of the (MSOM) according of the different values product.

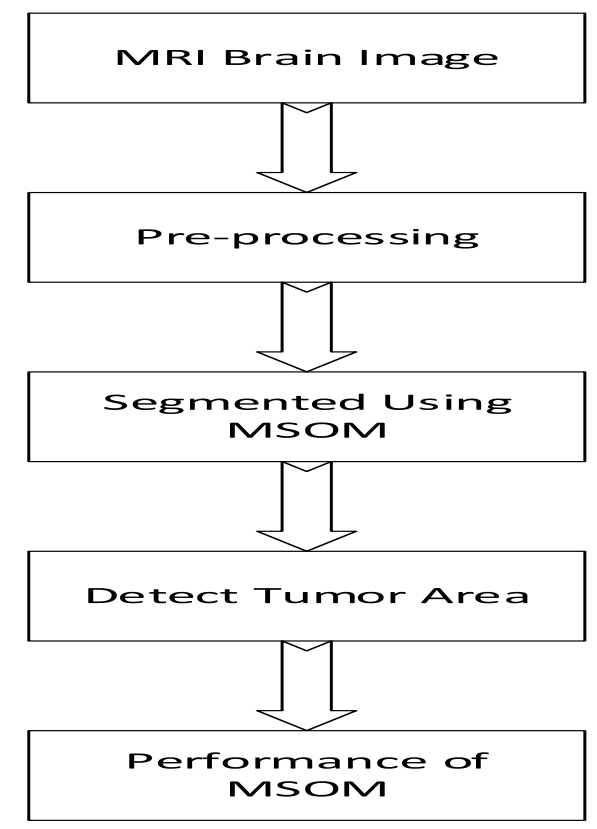

Figure.2. Flowchart explaining the (MSOM) algorithm framework

\subsection{Self-Organizing Map Algorithm}

Self-regulating maps (SOMs) has been proposed by Willshaw and Von Der Malsburg to visualize various biological phenomena observed in animals. In particular, they have discovered that some brain regions develop structures with different regions, each of which has a high sensitivity to a particular type of input pattern. 
The process behind such behavior is quite different because it is based on the rivalry between nerve units based on the principle called winner takes everything. During the training period, all units are excited with the same signal, but only one will produce the highest response. This module will automatically become a destination pool filter for this selected pattern. You will be introduced to the Kohonen model.

Kohonen SOM (also known as Kohonen network or Kohonen map) is simply represented as a twodimensional map (for example, a square matrix or any other rectangle), but three-dimensional surfaces, such as a ball or torus, are also possible Is the existence of a suitable scale). In your case, always refer to a square matrix, where each cell is a receptive neuron characterized by a tangential weight with dimensions of input patterns:

$$
X=\left\{\bar{x}_{1}, \bar{x}_{2}, \ldots, \bar{x}_{N}\right\} \text { where } \bar{x}_{i} \in \mathbb{R}^{n}
$$

During both training and working phases, the winning unit is determined according to a similarity measure between a sample and each weight vector. The most common metric is the Euclidean; hence, if you consider a bi-dimensional map $W$ with a shape $k \times p$ so that $W \in \mathrm{N} k \times p \times n$, the winning unit (in terms of its coordinates) is computed as follows:

$$
u^{*}=\operatorname{argmin}_{k, p}\left\|W[k, p]-\bar{x}_{x}\right\|_{2}
$$

As explained before, it is important to avoid the premature convergence because the complete final configuration could be quite different from the initial one. Therefore, the training process is normally subdivided into two different stages.

During the first stage, the duration is usually about $10-20 \%$ of the total number of frequencies (let us call this values max), and the correction is applied to the winning unit and its neighbors (computed by adopting a decaying radius).

However, during the second stage, the radius is set to 1.0 and the correction is applied only to the winning unit.

In this way, a larger number of possible configurations can be analyzed, and the configuration associated with the least error is automatically determined. The neighborhood can have different forms. Can be square (in 3D maps closed, no longer exists), or, more easily, a radial basis function can be used based on a significantly degraded distance weight:

$$
n(i, j)=e^{-\frac{\left\|u^{*}-(i, j)\right\|^{2}}{2 \sigma(t)^{2}}} \text { where } \sigma(t)=\sigma_{0} e^{-\frac{t}{r}}
$$

The relative weight of each neuron is determined by the $\sigma(t)$. The $\sigma 0$ function is the initial radius and $\tau$ is a time-constant that must be considered as a hyperactive parameter that determines the slope of the decaying weight. Suitable values are $\mathbf{5 - 1 0 \%}$ of the total number of iterations.

Adopting a radial basis function, it is not necessary to compute an actual neighbourhood because the multiplication factor $n(i, j)$ becomes close to zero outside of the boundaries. A drawback is related to the computational cost, which is higher than a square neighbourhood is (as the 
Abd El Kader Isselmou, Guizhi Xu, Shuai Zhang, Sani Saminu; Modified Self-Organizing Map Algorithm for Brain Tumor Detection and Analysis Using Magnetic Resonance Brain Images. Journal of Biomedical Engineering and Medical Imaging, Volume 6, No 2, April (2019), pp 27-34

\subsection{Morphological Operation}

Morphological image processing consists mainly of nonlinear processes that can relate to the shape or Image morphology features, such as borders, Skeletons etc. In morphological techniques, the image is Investigation by hiding a small template or a form called the elements of structure and operations are based on this element, which identifies the area of interest or neighborhood around Pixels.

Mathematical morphology is based on set theory operations, which are defined between set of points of an image called object and the kernel called structuring element, these are some basic morphological operations:

Dilation: Morphological expansion is a process consists of Find the maximum between pixels belongs to Window or window. Stretching removes the existing pixel noise Object area with object size increased, here input image $I$ with the size $G \times H$ and structuring element $B$ with the size $K \times L$, which defines the size of the window. Mathematically, it can be written as:

$$
[I \oplus B](w, l)=\max [I(w-u, l-v)|(u, v) \in B|
$$

Erosion: Morphological erosion is the operation, which consists of finding the minimum among the pixels belonging to the window. Erosion removes noise pixels, which are present in background with reducing size of object, here input image $I$ with the size $G \times H$ and $S E B$ with the size $K \times L$, which defines the size of the window. Mathematically, it can be written as:

$$
[I \ominus B](w, l)=\min [I(w+u, l+v) \mid(u, v) \in]
$$

\subsection{Accuracy Value}

Accuracy is the ability of the instrument to measure the accurate value. In other words, it is the closeness of the measured value to a standard or true value. The accuracy can be obtained by taking the small readings. The small reading reduces the error of the calculation. The accuracy of the system is classified in the following equation (6).

$$
\text { Accuracy }=\left(\frac{k}{m \times n}\right) \times 100
$$

\subsection{Dice Overlap Index (DOI) value}

It is expressed with the help of the value of the Jaccard index $J(A, B)$. DOI identify the purpose of overlap of the input image $(A)$, as well the resulting segmented image $(B)$.DOI mentions calculating in the equation (7).

$$
D(A, B)=2 \times \frac{J(A, B)}{1+J(A, B)}
$$

\subsection{Sensitivity value}

(Also called the true positive rate, the recall, or probability of detection in some fields) measures the proportion of actual positives that are correctly identified as such (e.g., the percentage of sick people who are correctly identified as having the condition), It is stated in Equation (8).

$$
O F=\frac{T P}{T P+F N}
$$




\subsection{Specificity value}

Also called the true negative rate) measures the proportion of actual negatives that are correctly identified as such (e.g., the percentage of healthy people who are correctly identified as not having the condition), specificity is shown in the Equation (9).

$$
\operatorname{Specificity}(\sigma)=\frac{T N}{T N+F P}
$$

\section{Results and Discussion}

In this work, we divided the results of experiments to the two sections principally as follows:

Section 1: Results processing of seven MR brain images using modified self-organizing map (MSOM) algorithm, (MSOM) algorithm detected tumor area clearly using FLAIR, $\mathrm{T} 1$, and $\mathrm{T} 2$ weight MR brain image as shown in the figure.3.

(a)

(b)

(c)

(d)

(e)

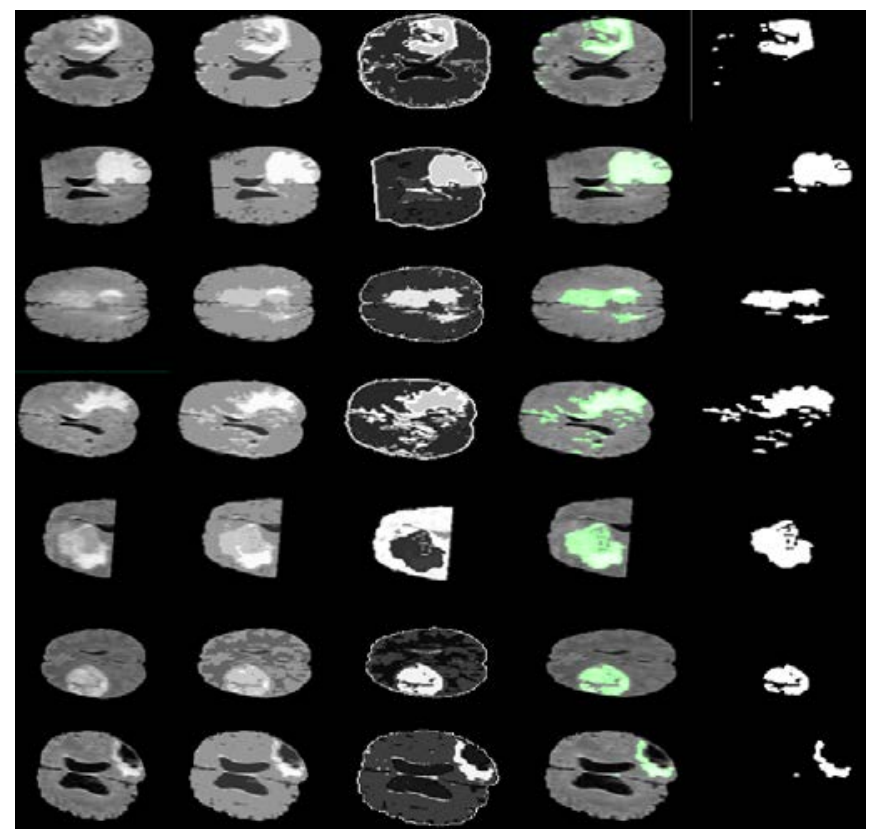

Figure.3 Results processing of the FLAIR, T1-T2 MR brain using modified self-organizing map (MSOM) algorithm

Image (a): FLAIR, T1-T2 MR Brain image or original image

Image (b): Quantization

Image (c): segmentation using modified self-organizing map (MSOM)

Image (d): Tumor area marked

Image (e): Tumor detection

The section 1 based on MR brain images processing to detect tumor area and it passed five important steps start it through input MR brain image and finished at detect tumor area applied (MSOM) algorithm, 
Abd El Kader Isselmou, Guizhi Xu, Shuai Zhang, Sani Saminu; Modified Self-Organizing Map Algorithm for Brain Tumor Detection and Analysis Using Magnetic Resonance Brain Images. Journal of Biomedical Engineering and Medical Imaging, Volume 6, No 2, April (2019), pp 27-34

the results of the processing and applied modified algorithm gave a best results and identify the tumor successfully using different types of MR brain images.

Section 2: Results performance of the modified self-organizing map (MSOM) algorithm, product the very good performance according of the values, the accuracy of detection is $81,79 \%$, nJaccard value is 63,966 , Ndice is 89,741 , sensitivity or true positive rate is $98,25 \%$, specificity or true negative rate is $98,25 \%$, recall is $98,25 \%$ and precision is $90,86 \%$ as describe in the figure 4 .

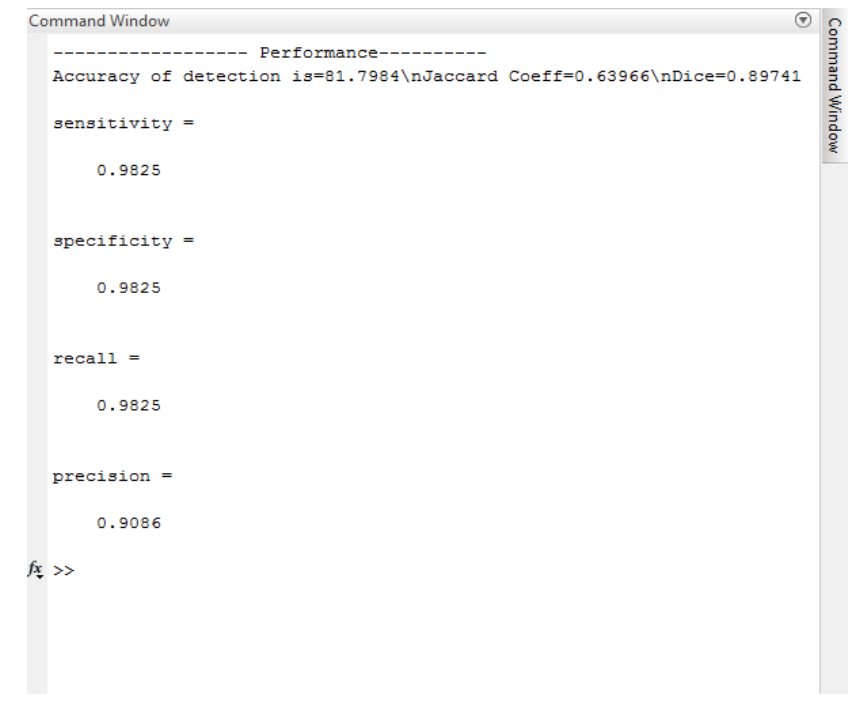

Figure.4 Performance of modified self-organizing map algorithm using values

\section{Conclusion}

In this work, we applied method based on modified self-organizing map algorithm (MSOM) for brain tumor detection and analysis, the objective of the modification it is to improve the performance of the method, and the modification results gave a best results in terms of tumor detection using MR brain images as describe in the figure 3 or performance of the modified self-organizing map (MSOM) algorithm, using different values as describe in the figure4, the results obtained from (MSOM) algorithm give the motivation to researchers in this area to continue the modification and improvement of the algorithm for more better results.

\section{ACKNOWLEDGE}

This work is supported by the National Natural Science Foundation of Hebei province under grant No: E2015202292 and No: E2015202050, high-level talent support project in Hebei province under grant No: C2015005012, Key research and development program under grant No: 15272002 and No: 15275704

\section{REFERENCES}

[1] Beutel, J., Kundel, H. L., \& Van Metter, R. L. (2000). Handbook of medical imaging: Physics and psychophysics (Vol. 1). Spie Press. 
[2] Esquenazi, Yoshua, Victor P. Lo, and Kiwon Lee. "Critical care management of cerebral edema in brain tumors." Journal of intensive care medicine 32.1 (2017): 15-24.

[3] Ortiz. J, Gorrizb J.M, Ramezb. J, Salas-Gonzalezb, D and Llamas-Elvirac J.M, Two Fully-unsupervised methods for MR brain image segmentation using SOM-based Strategies, Applied soft computing, (2013), 2668-2682.

[4] Logeswari. T and Karnan M, An improved implementation of brain tumor detection Using segmentation based on soft computing, Journal of Cancer Research and Experiment Oncology (JCREO(2010), 2(1) , 0614.

[5] Logeswari. T and Karnan M, an Improved Implementation of Brain Tumor Detection Using Segmentation Based on Hierarchical Self-Organizing Map, International Journal Of Computer Theory and Engineering, (2010).Vol. 2, No. 4.

[6] Yan Li and Zheru Chi, MR Brain Image Segmentation Based on Self-Organizing Map Network, International Journal of Information Technology, (2005) Vol. 11, No. 8.

[7] Guler, Demirhan. A and Karakis. R, Interpretation of MR images using self-organizing Maps and knowledge-based expert systems, Digital Signal Processing, 19 (2009), 668-677.

[8] Ong. S, Yeo. N, Lee. K, Venkatesh. Y and Cao. D, Segmentation of color images using A Two-stage selforganizing network, Image and Vision Computing, (2002), 20, 279-289.

[9] Alirezaie. J, M. Jernigan and C. Nahmias, Automatic segmentation of cerebral MR Images using artificial neural networks, IEEE Transactions on Nuclear Science, (1998), 45 (4) , 2174-2182.

[10] Sun. W, Segmentation method of MRI using fuzzy Gaussian basis neural network, Neural Information Processing, (2005), 8 (2), 19-24.

[11] Ipek Oguz and Milan Sonka, LOGISMOS-B: Layered Optimal Graph Image Segmentation of Multiple Objects and Surfaces for the Brain, IEEE Transactions on Medical Imaging, (2014), Vol. 33, No. 6, 1220 1235.

[12] Ayse Demirhan and Inan Guler, Combining stationary wavelet transform and Self-organizing maps for brain MR image segmentation, Engineering Applications of Artificial Intelligence, (2011), 24, 358-367. 